\title{
Consideration of limitations, which are formed by the input signal, on the phase error minimization process during carrier frequency tracking system of synchronization of radio technical device of communication
}

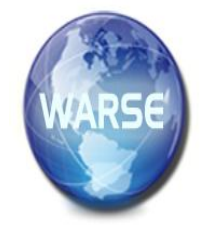

\author{
Oleksandr Turovsky ${ }^{*}$, Valerii Kozlovskyi ${ }^{2}$, Balanyuk Yuriy ${ }^{2}$, \\ Boiko Yuliia ${ }^{3}$, Lishchynovska Nataliia ${ }^{1}$ \\ ${ }^{1}$ State University of Telecommunications, Kyiv, Ukraine. \\ ${ }^{2}$ National Aviation University, Kyiv, Ukraine. \\ ${ }^{3}$ Taras Shevchenko National University of Kyiv, Kyiv, Ukraine. \\ * Corresponding author: s19641011@ukr.net
}

\begin{abstract}
The successful solution of the problem of maintaining high efficiency of communication and telecommunications systems largely depends on the quality of operation under the influence of various external and internal disturbances and noise of one of the main systems of their composition, namely combined phase synchronization systems. The effectiveness of the combined synchronization system generally depends on various external and internal factors and various restrictions imposed on the process of receiving and demodulating the input signal, the process of monitoring the carrier frequency of the system and minimizing the phase error variance. The presence of such limitations requires their analysis, evaluation and subsequent consideration in the process of synthesis of open communication of the combined synchronization system (CSS). The paper directly considers the issues of increasing the efficiency of the combined synchronization system by taking into account the limitations formed by the influence of a random input signal on the process of minimizing the phase error during the monitoring of the carrier frequency of the specified system. The results of the evaluation of the limitations formed by the influence of the random input signal on the process of minimizing the phase error during the monitoring of the carrier frequency of the CSS of the radio communication device are presented in the paper: in the presence of restrictions on any coordinate of the input signal, the effect of the introduction of an additional link of open communication in the synthesis of CSS is reduced and at some threshold values becomes " 0 " and the introduction of an additional link does not give the desired effect and becomes impractical; increasing the values of such constraints leads to an increase to the unit ratio of the corresponding functionalities for combined and closed synchronization systems.
\end{abstract}

Key words: carrier frequency synchronization, combined synchronization system, phase error variance, radio signal input signal limitation.

\section{INTRODUCTION}

The successful solution of the problem of maintaining high efficiency of communication and telecommunications systems largely depends on the quality of operation under the influence of various external and internal disturbances and noise of individual systems and devices that are part of them. As one of the main systems, which is a part of various radio engineering devices of communication, radar and control technology, as well as in the device of exact magnetic recording, the system of combined phase synchronization (KSS) is widely implemented. For example, in phase-coherent telecommunication and control systems, such systems are used to restore the carrier and clock frequencies and for coherent demodulation of analog and digital signals with angular modulation $[1,2]$.

The operation of synchronization systems is characterized by the direct influence of additive fluctuation noise, perturbation of useful angular modulation (in the case of carrier frequency filtering), phase and frequency jumps, etc. [2,3]. In some cases, it is necessary to ensure high accuracy of the specified system in steady and transient modes under the influence of these noises. And synchronization systems operating in such conditions should be characterized by low phase error variance and high speed.

Issues of improving the quality of the phase synchronization system are constant important scientific tasks and in some studies are solved by creating appropriate optimal schemes for its construction in the direction of minimizing the variance of the phase error and at the same time, ensuring high speed. It is obvious that these schemes solve the problem of minimizing the phase error through the development of scientifically sound optimal construction schemes that 
operate on the basis of developed mathematical models. These mathematical models must take into account both the parameters of the components of the scheme of construction of the synchronization system and the factors of external perturbations, as defined in $[4,5,6]$.

\subsection{Problem analysis}

In a number of works, namely in [6], mathematical dependences are presented that allow to determine the variance of the phase error under the influence of additive Gaussian noise. The evaluation of the phase error variance performed in this work showed that its reduction due to the introduction of open communication at a given effect depends on the noise signal level and the phase modulation index of the input signal. And when used as a simple open link functional link included in the circuit of the combined synchronization system in parallel, the minimum variance of the phase error when the noise level rises to a certain critical limit is limited and this open link loses its effectiveness.

Improving the efficiency of the combined synchronization system by synthesizing a complex open connection in the direction of further minimizing the variance of the phase error while maintaining a high level of dynamics of the system is considered in [7]. The mathematical dependences developed and presented in the work allow to carry out the synthesis of a complex open channel in the combined system synchronization system under the condition of minimizing the variance of the phase error, which includes two links of frequency discriminators. Due to the evaluation of the influence of the parameters of the complex link of open communication on the minimization of the phase error variance of the combined synchronization system from the signal level of the additive Gaussian noise it was shown that: the error variance value for the combined synchronization system is much smaller. has a limit to a certain critical limit, and open communication loses its effectiveness.

It is obvious that in addition to the factors identified in these works, there are a number of factors that can influence and limit the possibilities of synthesis of open communication of the above synchronization system, which was also noted in [3].

\section{MAIN MATERIAL}

2.1 Rationale for taking into account the limitations formed by the random input signal to minimize the variance of the phase error.

The effectiveness of the combined synchronization system depends in general on various factors, and directly on the level of additive interference at the input of the system, which is indicated in $[3,6,7]$.

In addition, there are other factors that reduce the positive effect of the introduction of additional complex open communication $[3,8,9]$.

In the synthesis of combined synchronization systems, a boosting signal is introduced into a closed loop, which affects the control element of the tuned generator, directly or through a low-pass filter (Fig. 1) [8,9]

Forcing signal is proportional to the derivative of the input signal. If the synchronization system is used to monitor the Doppler signal of the form (1) $[10,17]$.

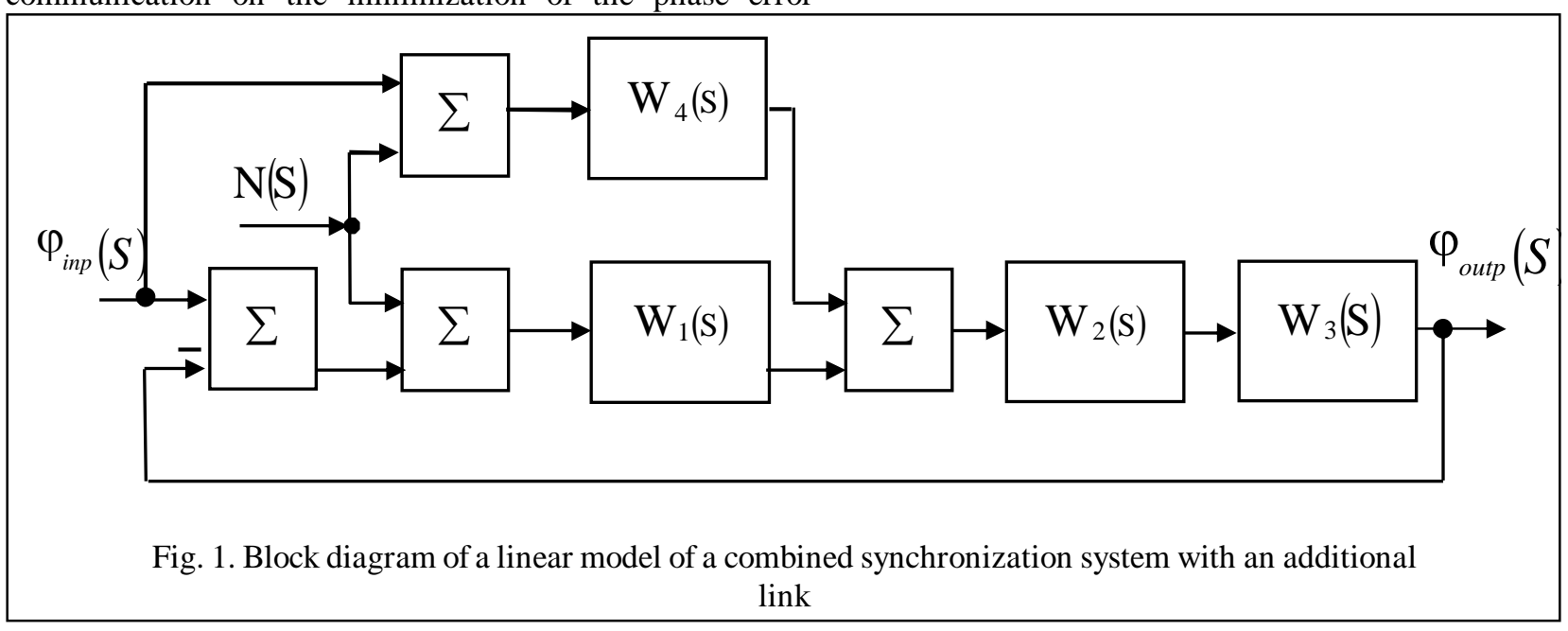

$$
\left.d(t)=\varphi_{0}+\sum_{r=0}^{N-1}\left(\Omega_{r} t^{r+1}\right)(r+1)\right)
$$

And $d(t)$ given by a function of polynomials type, the boost signal may include several derivatives of the input signal [8]. When angular demodulation against the background of noise, to reduce the variance of the phase error to the desired value. There is a need to introduce a number of derivatives in the boost signal. The total signal at the input of the control element can reach large peak values.

The adjustable generator is generally a nonlinear link with a nonlinearity of the saturation type [10]. The range of changes in its output frequency is limited by the characteristics of the control element. Changes in the second derivative of the output phase are limited by the inertia of the adjustable generator and the inertia of the filter if the boost signal is fed to the input of the low frequency filter (LFF). 
Therefore, the effect of the introduction of the boost signal decreases due to the presence of the above limitations and in the synthesis of CSS they must be taken into account [10]. In general, when forming the input effect on the system by a deterministic signal of the form (1) and if the input signal is random, it is necessary to take into account the restrictions on $\mathrm{N}$ derivatives of the input signal phase imposed on the dispersion of derivatives of the output signal phase $[3,10]$.

\subsection{Analysis of previous work}

A number of works are devoted to the issue of taking into account external and internal factors for the process of minimizing the phase error variance during carrier frequency monitoring when developing schemes for building a synchronization system and assessing the capabilities of these systems to minimize this phase error variance.

In the articles $[11,12]$ the possibilities for improving the quality of synchronization systems in the direction of minimizing the phase error are identified and substantiated in the class of combined synchronization systems (CCS). These systems can combine the principles of regulation of deviation and perturbation while ensuring the minimization of the phase error variance, which was defined as a promising method of constructing CSS. In turn, the issue of taking into account the limitations associated with the inertia of generators and LFF inputs to boost signals in these works is not discussed and not taken into account.

In [13] the peculiarities of the implementation of the carrier frequency recovery system with coherent demodulation of the signal with a continuous phase are shown. The scheme of construction of such a system of phase auto-tuning of frequency is essentially a combined synchronization system with feedback. The paper investigates the issue of practical implementation of this system on a modern element base. However, there is no research on the possibilities of the developed methods and the scheme of construction of the synchronization system to increase astatism, reduce the phase error dispersion when monitoring the carrier frequency and evaluate the proposed methods to reduce the phase error variance and impose various limitations on the evaluation process.

The authors of $[14,15]$ proposed a method of implementing a certain type of signal sequence synchronization in CSS, which expands under conditions of significant excess of the noise level over the level of the information signal. For synchronization the service channel which works on one frequency with information is used. Channel distribution is performed during the formation of signals of quadrature channels: in-phase channel is used to generate a phase-manipulated signal with spectrum expansion, quadrature channel is used to transmit a clock signal. The possibility of minimizing the phase error by taking into account the limitations imposed by the inertia of generators and LFF synchronization system is not considered in this paper.

In the articles [16] presents a new direct sequence modulation scheme for distributed spectrum communication systems, which is defined as delay and addressing modulation (DADS). The proposed scheme is easy to implement and does not require alignment of the input signal code at its input, which makes it the most optimal for the transmission of short signals. The paper does not disclose the type of scheme in relation to which the conclusions were substantiated, as well as there is no question of increasing the order of astatism and taking into account the limitations formed by the peculiarities of the scheme defined in this paper.

Some studies on the possibilities of minimizing the variance of the phase error in the open-loop CSS and one of the options for constructing the CSS, which has such a combination along with the high speed of the system are presented in [17]. This paper develops and presents a mathematical model of KSS with an open connection of a certain type and evaluates its ability to minimize the phase error in the order of astatism not higher than the second. It is directly established that the open channel, made in the form of parallel (serial) connection of two links of the frequency discriminator with the proposed transfer function, allows to increase the order of astatism to the third and higher order and does not affect the stability of the system. The paper shows that the effect on the variance of the phase error of the synchronization system, as substantiated in this paper, can be achieved by changing the parameters of the open link of the synchronization scheme of the system. The issue of minimizing the phase error under the influence of the constraints imposed by the inertia of generators and LFF synchronization system is not considered in this paper.

Thus, taking into account the limitations formed by the influence of a random input signal on the inertia of adjustable generators and on the inertia of the low-pass KSS in the synthesis of its open communication is an urgent scientific problem to which this work is devoted.

\subsection{Statement of the research problem}

Consider the solution of the scientific problem of minimizing the root mean square error when the signal phase is modulated by a random process $(2)[18,19]$ :

In the future, we will accept the modeling signal $m(t)$ in the form of two types: with the "maximally flat (butterworth) shape of the spectrum" and asymptotic Gaussian processes. Their energy spectra can be described according to the following expressions $[10,18]$ :

$$
G_{2}(\omega, n)=\frac{K_{2}(n)}{\left[1+\left(\frac{\omega}{\sqrt{n} \omega_{c}}\right)^{2 n}\right]^{n}}, n=1,2,3 \ldots,
$$

where $K_{2}(n)=\frac{(4 \pi)}{\left[\sqrt{n \omega_{c}}\right]}, \sin c=\sin \frac{x}{x}$,

$\omega_{c}-$ frequency corresponding to half the power. 
The following spectra are characteristic of a wide class of signals used in communication [18]:

$$
G_{1}(\omega, 1)=G_{2}(\omega, 1)=G(\omega, 1),
$$

when $n=1$ they are the same.

Consider the possibility of reducing the root mean square error (RMSE), which is associated with the minimum limiting variance of the phase error when tracking the carrier frequency in the CSS.

When $n=1$ the dispersion of the first and second derivatives of the original phase restrictions are imposed [19]:

$$
\sigma_{\ddot{\text { pout }}}^{2}<Q_{1}, \sigma_{\ddot{\varphi} \text { out }}^{2}<Q_{2}
$$

Under such conditions, the additional signal on the open link will have a smaller effect than in the system without restrictions. And with some constraint limits, additional open communication becomes ineffective. [6,7].

2.4 Development of functional dependencies to take into account the limitations formed by the input signal to the process of minimizing the variance of the phase error

Let us estimate the influence of the constraints imposed on the original coordinate of the synchronization system and on the magnitude of the variance of the phase error in the combined synchronization system with deviation adjustment. To do this, we derive the functional dependence that connects the variance of the phase error with the variance of the first and second derivative of the original coordinate, taking into account the limitations in CSS $[10,19]$ :

$$
F=I_{\varphi}+\rho_{1} I_{\ddot{\varphi} \text { out }}+\rho_{2} I_{\ddot{\text { 甲out }}},
$$

when: $\rho_{1}, \rho_{2}-$ Lagrange multipliers, $I_{\varphi}=\sigma_{\ddot{\varphi} o u t}^{2}$, $I_{\ddot{\varphi} \text { out }}=\sigma_{\ddot{\varphi} \text { out }}, I_{\ddot{\varphi} \text { out }}=\sigma_{\ddot{\text { pout }}}^{2}$.

The first component determines the minimum value of the phase error and is determined by expression (4):

$$
\sigma_{\varphi K \min }^{2}=\sigma_{\varphi 3}^{2}-\Delta \sigma_{\varphi}^{2},
$$

when: $\quad \sigma_{\varphi 3}^{2}=N_{2}\left(a_{0} \beta_{11}-a_{0} \beta_{11}\right)-N_{12}\left(\alpha_{21}-\alpha_{23} \beta_{21}\right)$, $\Delta \sigma_{\varphi}^{2}=\left(\alpha_{21} N_{1} K_{3}-a_{2} N_{2} K_{1}^{2} K_{3}\right) / 2>0$.

The first component can also be defined by an expression which after simple transformations can be written in the form $[19,20]$.

$$
I_{\varphi}=A_{1} K_{4}^{2}+A_{2} K_{4}+A_{3},
$$

when: $A_{1}=-\left(8 \alpha_{21} A_{0}^{2} K_{3}^{2}\right) / C_{0} \quad, \quad A_{2}=\left(26 \alpha_{21} A_{0} K_{3}\right) / C_{0}$, $\mathrm{A}_{3}=-\mathrm{B}\left(\alpha_{21}+\alpha_{23} \mathrm{~T}_{4}^{2}\right) / \mathrm{C}_{0}$
$\mathrm{C}_{0}=2\left(\alpha_{20} \alpha_{21}^{2}+\alpha_{24} \alpha_{21}^{2}-\alpha_{21} \alpha_{22} \alpha_{23}\right)$

Components $I_{\dot{\varphi} \text { outp }}$ and $I_{\dot{\varphi} \text { inpt }}$ find from the ratios

$$
\begin{gathered}
I_{\dot{\varphi} \text { int } p}=\frac{1}{2 \pi} \int_{-\infty}^{\infty}\left|W_{\varphi K}(j \omega)(j \omega)\right|^{2} G_{M}(\omega) d \omega \leq \mathrm{Q}_{1}, \\
I_{\ddot{\varphi} \text { outp }}=\frac{1}{2 \pi} \int_{-\infty}^{\infty}\left|W_{\varphi K}(j \omega)(j \omega)^{2}\right|^{2} G_{M}(\omega) d \omega \leq \mathrm{Q} 2
\end{gathered}
$$

Substituting in the above ratios expressions for transmission and energy functions ${ }^{\wedge}$

$$
\begin{gathered}
W_{\varphi K}(S)=\left(b_{0} S^{2}+b_{1} S\right) /\left(a_{0} S^{2}+a_{1} S+a_{2}\right) \\
=D_{\varphi K}(S) / F_{3}(S) \\
G_{2}(w, n)=K_{2}(n) /\left[1+\left(\omega / \sqrt{n} \omega_{c}\right)^{2 n}\right]^{n}, n-1,2,3 \ldots
\end{gathered}
$$

and defining integrals by expression (9) as the Perseval integral [19]:

$$
I_{n}=\frac{1}{2 \pi} \int_{-\propto}^{\propto} \frac{M(j \omega)}{\mathrm{H}(j \omega)-\mathrm{H}(j \omega)} d \omega,
$$

when: $\mathrm{H}(j \omega)=\sum_{i=0}^{n} \alpha_{i}(j \omega)^{n-i}, \mathrm{M}(j \omega)=\sum_{i=0}^{n-1} \beta_{i}(j \omega)^{2(n-i-1)}$.

We will receive

$$
\begin{aligned}
& I_{\dot{\varphi} \text { out }}=\frac{K_{m}^{2} \theta}{2 \pi} \int_{-\infty}^{\propto}\left|\frac{\beta_{0}(j \omega)^{2}+\beta_{1}(j \omega)}{a_{10}(j \omega)^{2}+a_{11}(j \omega)+a_{12}}\right|^{2} \frac{d \omega}{\left(\omega_{c}^{2}+\omega^{2}\right)^{2}}= \\
& =\frac{K_{m}^{2} \theta}{2 \pi} \int_{-\propto}^{\propto} \frac{\beta_{10 \dot{\varphi}}(j \omega)^{6}+\beta_{11 \dot{\varphi}}(j \omega)^{4}+\beta_{12 \dot{\varphi}}(j \omega)^{2}+\beta_{13 \dot{\varphi}}}{\left|\alpha_{20}(j \omega)^{4}+\alpha_{21}(j \omega)^{3}+\alpha_{22}(j \omega)^{2}+\alpha_{23}(j \omega)+\alpha_{24}\right|^{2}} d \omega= \\
& =\frac{K_{m}^{2} \theta}{2} \frac{\alpha_{21} \beta_{12 \dot{\varphi}}-\alpha_{23} \beta_{11 \dot{\varphi}}}{\alpha_{20} \alpha_{23}^{2}+\alpha_{24} \alpha_{21}^{2}-\alpha_{21} \alpha_{22} \alpha_{23}}=B_{1} K_{4}^{2}+\beta_{1} K_{4}+\beta_{3}
\end{aligned}
$$

when: $\beta_{10 \dot{\varphi}}=\beta_{13 \dot{\varphi}}=0, \quad \beta_{11 \dot{\varphi}}=\mathrm{B}_{0}^{2}, \quad \beta_{12 \dot{\varphi}}=\mathrm{B}_{1}^{2}$,

$$
\begin{aligned}
& \mathrm{B}_{1}=-\left(\alpha_{23} \mathrm{~A}_{0}^{2} \mathrm{~K}_{3} \mathrm{~K}_{\mathrm{m}}^{2} \mathrm{~B}\right) / \mathrm{C}_{0} \\
& B_{2}=-2\left(\alpha_{23} A_{0}^{2} K_{1} K_{3} K_{m}^{2}{ }^{2}\right) / C_{0} \\
& B_{3}=-\left(\alpha_{21}+\alpha_{23}\right)\left(A_{0} K_{1} K_{3}^{2} \theta\right) / C_{0} .
\end{aligned}
$$




$$
\begin{aligned}
& I_{\dot{\varphi} \text { out }}=\frac{K_{m}^{2} \theta}{2 \pi} \int_{-\propto}^{\infty}\left|\frac{B_{0}(j \omega)^{3}+B_{1}(j \omega)^{2}}{a_{0}(j \omega)^{2}+a_{1}(j \omega)+a_{2}}\right|^{2} \frac{d \omega}{\left(\omega_{c}^{2}+\omega^{2}\right)^{2}}= \\
& =D_{1} K_{4}^{2}+D_{2} K_{4}+D_{3}
\end{aligned}
$$

when:

$$
\begin{aligned}
& D_{1}=-\left[\left(A_{0} K_{3}\right)^{2}\left(\alpha_{22} \alpha_{23}-\alpha_{21} \alpha_{24}\right) K_{m}^{2} \theta\right] /\left(\alpha_{20} C_{0}\right), \\
& D_{2}=-\left[2 K_{1}\left(A_{0} K_{3}\right)^{2}\left(\alpha_{22} \alpha_{23}-\alpha_{21} \alpha_{24}\right) K_{m}^{2} b\right] /\left(\alpha_{20} C_{0}\right), \\
& D_{3}=-\left[\left(A_{0} K_{1} K_{3}\right)^{2}\left(\alpha_{22} \alpha_{23}-\alpha_{21} \alpha_{24}+\alpha_{20} \alpha_{23}\right) K_{m}^{2} b\right] /\left(\alpha_{20} C_{0}\right) .
\end{aligned}
$$

Then the functional (3) for the synchronization system with combined control can be written as follows [19, 21]:

$$
\begin{aligned}
& F_{K}=A_{1} K_{4}^{2}+A_{2} K_{4}+A_{3} \\
& +\rho_{1}\left(b_{1} K_{4}^{2}+b_{2} K_{4}+D_{3}\right) \\
& +\rho_{2}\left(b_{1} K_{4}^{2} b_{2} K_{4}+D_{3}\right)
\end{aligned}
$$

A similar functionality for a system with the principle of regulation by deviation is obtained from inequality (10) when substituting in the last value $K_{4}=0 .[19,21]$ :

$$
F_{3}=A_{3}+\rho_{1} B_{3}+\rho_{2} D_{3}
$$

The optimal value of the parameter of the additional connection, which can minimize the functional (10), we find if we take the derivative $F_{K}$ of the parameter and equate it to "0".

$$
K_{4 O P T}=-\frac{A_{2}+\rho_{1} b_{2}+\rho_{2} D_{2}}{2\left(A_{1}+\rho_{1} b_{2}+\rho_{2} D_{1}\right)}
$$

To determine the Lagrange multiplier, substitute the value $K_{4 O P T}$ from (12) in the equation of constraints (5) and obtain the following system of equations $[19,20,21]$ :

$b_{1}\left[\frac{A_{2}+\rho_{1} b_{2+} \rho_{2} D_{2}}{2\left(A_{1}+\rho_{1} b_{1+} \rho_{2} D_{1}\right)}\right]^{2}-b_{2} \frac{A_{2}+\rho_{1} b_{2+} \rho_{2} D_{2}}{A_{1}+\rho_{1} b_{1+} \rho_{2} D_{1}}+b_{3}=Q_{1}$

The solution of which makes it possible to determine the value $\rho_{1}, \rho_{2}$.

\subsection{Estimation of the limitations formed by the input signal to minimize the variance of the phase error}

Based on expressions (10), (11), (12) we will perform mathematical modeling and construct and refine graphs of dependences $F_{K} / F_{3}=f_{1}\left(F_{4}\right), K_{4}$ OPT $=f_{2}(\rho)$ [21]

The values of the parameters of the system and the input signal are taken in the values:
$T_{1}=0.01 \mathrm{c}, T_{2}=0.1 \mathrm{c}, K_{1}=K_{3}=\mathrm{A}_{0}^{2}=1.0$

Relevant graphs, with clarifications on the calculation and simulation conditions are presented in Fig. 2 and Fig. 3 [19, 21].

The analysis shown in Fig. 2 and Fig. 3 dependencies shows that if the system has restrictions on any coordinate, the effect of the introduction of an additional link is reduced.

In this case, with increasing constraints, the ratios of the corresponding functionalities for CSS and the synchronization system is closed approach one - Fig.2. In addition, from the graphs of Fig. 3 it can be seen that with increasing constraints imposed on the original coordinate, the parameter of the numerator of the additional open link decreases and at some threshold values becomes " 0 ". That is, the introduction of an additional link does not give the desired effect and becomes impractical.

Thus, for the considered system, the limit value of the Lagrange factors characterizing the constraints imposed on the variance of the first and second derivatives of the original coordinate will be $\rho_{1}=0.74 \times 10^{-1}$, and $\rho_{2}=0.87 \times 10^{-3}$.
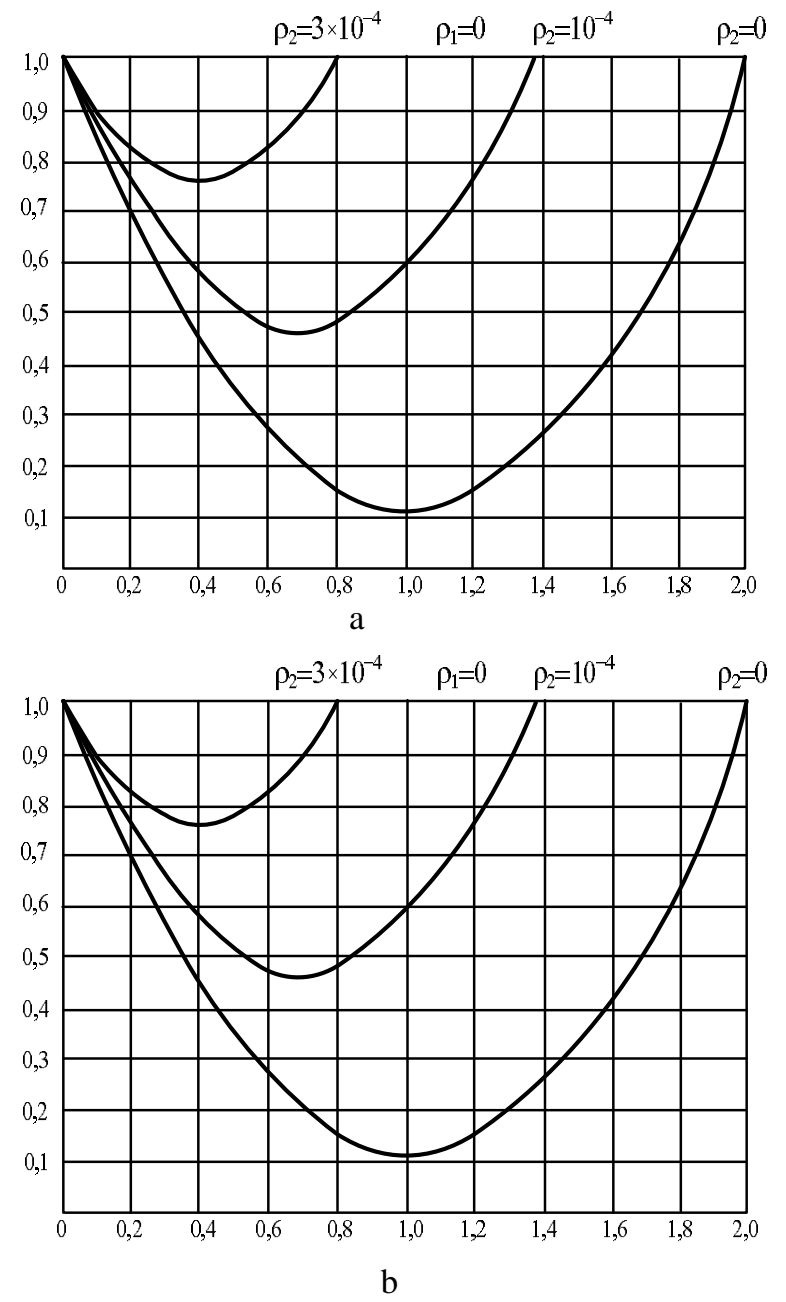

Fig. 2. Graph of dependence $F_{K} / F_{3}=f_{1}\left(K_{D}\right)$

Problems of signal transmission in modern satellite systems are determined by certain features of both the construction of 
the system itself and the problems of signal reception and transmission.

The existing energy of the satellite communication channel causes an urgent need for coherent signal processing and the use of powerful noise-tolerant coding. The noise-tolerant coding system is an integral part of the satellite modem. The vast majority of systems use high-precision encoding with Viterbo decoding and cascading codes. Widespread are turbo codes and codes with low density of parity checks [22, 23].

It should be noted that in general, when receiving the input signal by the synchronization system, the actual problem is to reduce the effect of noise interference in order to increase the signal-to-noise ratio (SNR). This problem is also relevant for other technical systems operating under various influencing factors. One of the methods of reducing the impact of noise interference at the input of the synchronization system is quite illustratively presented in $[24,25]$.
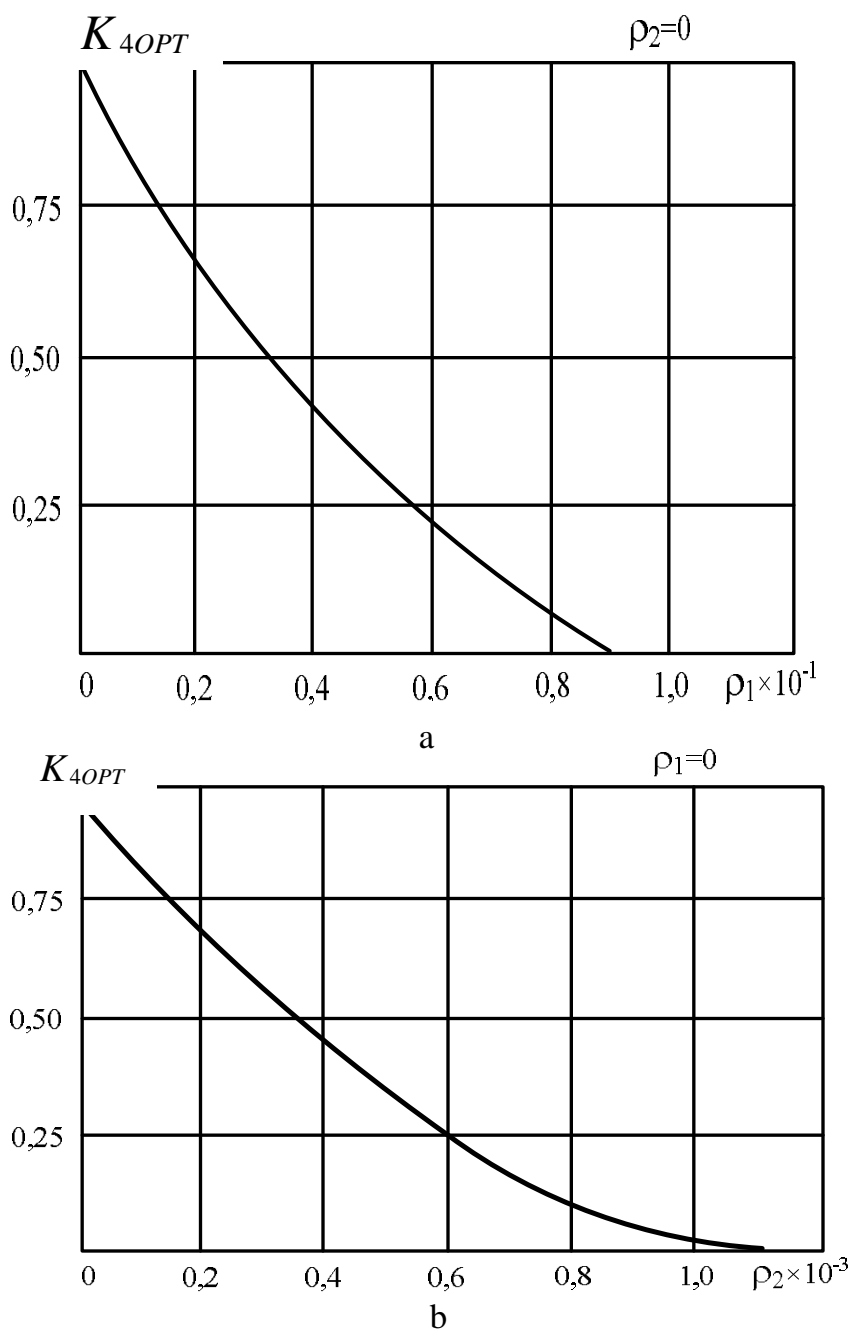

Fig. 2. Graph of dependence

$$
\mathbf{a}-K_{4 O P T}=f_{1}\left(\rho_{1}\right), \mathbf{b}-K_{4 O P T}=f_{2}\left(\rho_{21}\right),
$$

The scientific article [25] is devoted to orthogonal Lager filtering of noise processes, which are described by linear random processes. The proposed filtration method makes it possible to reduce the influence of noise interference, which is described by stationary linear random processes, during the operation of correlation systems. The idea of this method is to use orthogonal Lagerr filters as input links of the correlation system. This method can be used in further studies to increase the efficiency of the process of minimizing the variance of the phase error in the process of monitoring the carrier frequency.

It should be noted that the quality of the carrier frequency estimate is significantly affected by the parameters of the noise environment, which can be formed by various environmental factors, among which are both external and internal noise. Among the list of internal noises of some interest in the process of frequency estimation may take into account the internal noise associated with changes in nonlinear properties of composite materials of the synchronization system under the influence of increasing number of additional tracks of charge carriers due to decay in the material structure of radioisotope inclusions [26]. This can affect the growth of the internal noise of the synchronization system and requires its consideration in the development of advanced systems.

\section{CONCLUSION}

Mathematical dependences are developed in the paper and on the basis of their application the results are obtained, which allow to estimate the limitations formed by the influence of random input signal on minimization of phase error in the process of monitoring the carrier frequency of the combined synchronization system.

The results of the estimation of the limitations formed by the influence of the random input signal on the minimization of the phase error in the process of monitoring the carrier frequency of the combined synchronization system of the radio communication device are presented in the paper.

- in the presence of restrictions on any coordinate of the input signal, the effect of the introduction of an additional link of open communication in the synthesis of KSS is reduced and at some threshold values becomes " 0 " and the introduction of an additional link does not give the desired effect and becomes impractical;

- increasing the values of such constraints leads to an increase to the unit ratio of the corresponding functionalities for combined and closed synchronization systems.

\section{REFERENCES}

1. Horbatyy I.V. Systems of remote sensing of the Earth from space: monograph, SPOLOM, Lviv, 2011.612 p.

2. Boiko, Juliy., Pyatin Ivan, Eromenko Oleksander, Barabash Oleg "Methodology for Assessing Synchronization Conditions in Telecommunication Devices", Advances in Science, Technology and Engineering Systems Journal, No. 5, pp. 320-327, 2020. https://doi.org/10.25046/aj050242.

3. Boiko J. "Increasing the noise immunity of signal processing units of telecommunications on the basis of the modified synchronization schemes", Visnyk NTUU 
KPI Seriia - Radiotekhnika Radioaparatobuduvannia, No. 61, pp. 91-107, $2015 . \quad$ doi: 10.20535/RADAP.2015.61.91-107.

4. Oleksandr Turovsky, Liubov Berkman, Oleksandr Drobyk, Andrii Zakharzhevskyi, Oleksiy Khakhlyuk "Determination of the lower border of Cramer-Rao for evaluation of the carrier frequency of the radio technical communication channel signal", International Journal of Advanced Trends in Computer Science and Engineering, Vol. 9, No.4, pp. 5838 - 5845, 2020. https://doi.org/10.30534/ijatcse/2020/243942020.

5. Oleksandr Turovsky, Oleksandr Drobyk, Anatoliy Makarenko, Oleksiy Khakhlyuk "Estimates of the carrier frequency of the signal received by the satellite communication system in package mode", International Journal of Advanced Trends in Computer Science and Engineering, Vol.9, No.3, pp.3 223 - 3228, 2020. https://doi.org/10.30534/ijatcse/2020/115932020.

6. Turovsky O.L. "Estimation of the possibilities of the combined synchronization system with open-link to minimize the dispersion of the phase error when tracking the carrier frequency under the conditions of the influence of additive noise", Technology audit and production reserves. Vol.3, No.4, pp. 16-22, 2020. DOI: 10.15587/2706-5448.2020.210242.

7. Turovsky O.L. "Synthesis of a complex open communication in a synchronization system under the condition of minimizing the phase error variance during carrier frequency tracking", Visnyk of Khmelnytsky National University, No.3, pp. 46-54, 2020.

8. Safaryan O.A. "Method for estimating the frequency of generators in conditions of unpredictable changes in the duration of the measurement interval", Vestnik DSTU, Vol. 14, No.4 (79), pp. 142-149. 2014.

9. Gabrielian D.D., Yengibaryan I.A., Safaryan O.A. "New method of generator frequency stabilization", REDS: telecommunication devices and systems, Vol. 4 . No.1, pp.30-34, 2014.

10. Lyons R.G Understanding Digital Signal Processing, Prentice Hall, Boston, 2010. 992 p.

11. Boyko Y.M. "Evaluation of quality indicators of devices for synchronization of signals of telecommunications", Bulletin of Khmelnitsky National University, No.1, pp. 144-155, 2015.

12. Scheers B. "A Modified Direct-Sequence Spread Spectrum Modulation Scheme for Burst Transmissions", Military Communications and Information Systems Conference (MCC'2010), Wroclaw, Poland, September 27-28 2010, pp. 366-373.

13. Shakhtarin B.I. Analysis of synchronization systems in the presence of interference. 2 nd ed., Rev. and add, Hot line - Telecom, Moscow, 2016, 360 p.

14. Kay S. “Accurate Single Frequency Estimator", IEEE Trans. Acoust. Speech, Signal Processing, Vol.37, No.12, pp. 1987-1990, 1989. doi: 10.1109/29.45547.
15. Tikhomirov A. V. "Synchronization in systems with direct spreading of the spectrum", Engineering Bulletin of the Don, No.9, pp. 31-35, 2019.

16. Le Nir V. "Blind CP-OFDM and ZP-OFDM Parameter Estimation in Frequency Selective Channels", Wireless Com Network, No.315765, 2009. https://doi.org/10.1155/2009/315765

17. Oleksandr Turovsky, Khlaponin Y., Muhi-Aldin H. M. et al, "Combined system of phase synchronization with increased order of astatism in frequency monitoring mode", CEUR Workshop Proceedings, Vol.2616, Session.1, pp.53-62, 2020.

18. Sklar B. Digital Communications: Fundamentals and Applications. Second Edition. Prentice Hall, Boston, 2017. 1104 p.

19. Horowitz P. and Hill W. The Art of Electronics, Cambridge University Press, Cambridge, 2015. 1220 p.

20. Polikarovskikh A.I. "Modern reference generators for frequency and signal synthesis systems", Bulletin of the Voronezh State Technical University, Vol.10, No.4. pp. 100-108, 2014.

21. Nasir A., Durrani S. and Kennedy R. "Particle filters for joint timing and carrier estimation: Improved resampling guidelines and weighted bayesian cramer-rao bounds", IEEE Trans. Commun, No.60(5), pp.1407-1419, 2012.

22. Shestakov A.L., Semenov A.S., Ibrieva O.L. "Estimation of the carrier frequency of a random sequence of pulses by the Proni method", Bulletin of SUSU, No.37 (170), pp. 106-115, 2009.

23. Sana Fathima, Sudershan Reddy Kotla, Sameeha Fahmeen, Quddusa Sultana, Desireddy Krishna Reddy. "Estimation and Analysis of Instrumental Biases for GPS and NavIC Satellites and Receivers", International Journal of Advanced Trends in Computer Science and Engineering. Vol.8, pp. 391-394, 2019, doi: 10.30534/ijatcse/2019/5981.42019.

24. Kozlovskyi V., Scherbak L, Martyniuk H., Balanyuk Y. Boiko, Y. "Applying an adaptive method of the orthogonal laguerre filtration of noise interference to increase the signal/noise ratio", Eastern-European Journal of Enterprise Technologies, No.2(9-104), pp.14-21,

2020. DOI: 10.15587/1729-4061.2020.201397.

25. Kozlovskyi V., Korzh R., Petrovska S., Boiko Y., Yakoviv I. "Low-Frequency Schemes of Substitution of Segments Inhomogeneous Transmission Lines", 3rd International Conference on Advanced Information and Communications Technologies, AICT 2019 Proceedings, $2019 . \quad$ pp. 80-83, DOI: 10.1109/AIACT.2019.8847844.

26. Savchenko Vitalii, Vorobiov Oleh, Tkalenko Oksana et all "Influence of the Composite Materials Nonlinear Properties with Radioisotope Inclusions on Reflected Radiation", International Journal of Advanced Trends in Computer Science and Engineering, Vol.8, pp. 2716-2720, 2019, doi:10.30534/ijatcse/2019/05862019. 